\title{
Stimulus conflict predicts conflict adaptation in a numerical flanker task
}

\author{
WIM NOTEBAERT and TOM VERGUTS \\ Ghent University, Ghent, Belgium
}

\begin{abstract}
Conflict monitoring theory states that response conflict triggers conflict adaptation, resulting in reduced congruency effects after response-incongruent trials (Botvinick, Braver, Barch, Carter, \& Cohen, 2001). Verbruggen, Notebaert, Liefooghe, and Vandierendonck (2006) observed conflict adaptation after stimulus-incongruent trials without any response conflict. In this study, we further explore the hypothesis that stimulus conflict is an important trigger for conflict adaptation. We propose a measure for stimulus conflict that adequately explains the data of Verbruggen et al. and new data from a numerical flanker task. We conclude that stimulus conflict and response conflict have dissociable effects on behavior: Whereas response conflict is a good predictor of response times, stimulus conflict is a better predictor of the adaptation effect.
\end{abstract}

The central issue in the study of cognitive control is how the cognitive system is capable of monitoring and controlling information processing. One influential answer to this question is provided by conflict monitoring theory (Botvinick, Braver, Barch, Carter, \& Cohen, 2001), which describes how conflict is detected and how adjustments are made after conflict detection. In a variety of tasks, congruency effects are smaller after incongruent than after congruent trials. Conflict monitoring theory states that conflict detection leads to increased cognitive control, which reduces the influence of irrelevant information on the next trial. Assume that participants respond to a central arrow by pressing a left or right response key. Trials can be either congruent (low conflict-e.g., $>>>$ ) or incongruent (high conflict-e.g., $><>$ ). The flanker effect is calculated as the difference in response time (RT) between congruent and incongruent trials. Gratton, Coles, and Donchin (1992) demonstrated that this flanker effect was reduced when the previous trial was incongruent (high conflict). This was replicated in the Simon task (Stürmer, Leuthold, Soetens, Schröter, \& Sommer, 2002), the Stroop task (Kerns et al., 2004), and prime-target interference (Kunde, 2003). Consequently, the reduction of conflict after conflict situations seems to be a general phenomenon, even when the design is carefully controlled for confounding bottom-up repetition effects. Hommel, Proctor, and Vu (2004); Mayr, Awh, and Laurey (2003); and Notebaert, Soetens, and Melis (2001) raised this concern, and Kerns et al. (2004); Notebaert, Gevers, Verbruggen, and Liefooghe (2006); and Wühr and Ansorge (2005)

We thank Frederick Verbruggen, Wim Gevers, Eric Soetens, and André Vandierendonck for their comments and suggestions on a draft version of the manuscript. Correspondence concerning this article should be addressed to W. Notebaert, Experimentele Psychologie, Henri Dunantlaan 2, B-9000 Ghent, Belgium (e-mail: wim.notebaert@ugent.be). demonstrated that even after this confound is controlled for, conflict adaptation is observed.

An essential aspect of conflict monitoring theory is the description of how conflict is measured. In the original theory, conflict is conceptualized as the simultaneous activation of competing responses. When two response candidates (e.g., left response and right response) are both activated, there is response conflict. Formally,

$$
\text { Response conflict }=-\sum_{i j} z_{i} z_{j} w_{i j}
$$

where $z_{i}$ and $z_{j}$ are the activation values of the different response nodes and $w_{i j}$ represents the (inhibitory) connection weight between these response nodes.

Conflict can be defined at levels lower than the response level, and in particular, at the stimulus level. Unfortunately, in most studies response conflict has been confounded with stimulus conflict, making it unclear to what extent stimulus conflict is involved. Verbruggen, Notebaert, Liefooghe, and Vandierendonck (2006) compared the adaptation of the flanker effect after stimulus and response conflict. They mapped six colors on three responses. Trials in which a target is flanked by the same color are congruent $(\mathrm{Co})$ trials. When the flanker is different but associated with the same response, the conflict is located solely at stimulus level and the trial is stimulus incongruent (SI). When the flanker is a different color and this color is also associated with a different response, there is both stimulus conflict and response conflict, and the trial is response incongruent (RI). The data showed that there was conflict adaptation after trials with only stimulus conflict. There was no difference between the adaptation after SI trials and after RI trials. This suggests that stimulus conflict is sufficient to trigger adaptation. Nevertheless, the RTs were determined by the response conflict and not by stimulus conflict. The effect of stimulus conflict on RTs in many-to-one mappings is not clear, 
however. The difference between SI and Co trials is sometimes nonexistent (Verbruggen et al., 2006), sometimes significant but much smaller than the difference between SI and RI (van Veen, Cohen, Botvinick, Stenger, \& Carter, 2001), or as large as the difference between SI and RI (van Veen \& Carter, 2005).

To clarify the respective roles of stimulus conflict and response conflict in the adaptation of the flanker effect, it is useful to have a quantitative measure of stimulus conflict in addition to that of response conflict. This measure must be maximal when all stimuli are equally activated and minimal when only one stimulus is activated. Possibly the simplest measure that fulfills these conditions is the reciprocal of the standard deviation $(S D)^{1}$ of the input activation values-that is,

$$
\text { Stimulus conflict }=\frac{1}{S D} \text {. }
$$

For example, when there are two stimuli, there are two input nodes, each coding for one stimulus. When only the first stimulus is presented, the input activation pattern is $(1,0)$, so $S D=1 / 2$ and stimulus conflict $=2$. On the other hand, when both stimuli are equally active, the input pattern is $(0.5,0.5)$ and stimulus conflict equals $1 / 0=+\infty$. To account for scaling factors, one could define stimulus conflict as $1 /$ (coefficient of variation $)=1 /(S D /$ mean $)$; application of this equation leads to results very similar to those described here.

We implemented a neural network with three stimulus nodes and two response nodes in order to test the predictions of both measures. A schematic overview of the model is shown in Figure 1A. At the input level, each of three possible stimuli has its own dedicated input node. At the response level, each of two possible responses has its own response node. In the stimulus configuration shown in Fig- ure $1 \mathrm{~A}$, stimulus conflict would be $1 / S D(1,0.5,0)$, or approximately 2.45 . (See the Appendix for formal details.)

With this simple setup, we were able to distinguish Co, SI, and RI trials (as in Verbruggen et al., 2006). We calculated stimulus conflict, response conflict, and predicted RTs according to the model. Adaptation (the influence of conflict on trial $n-1$ on the RTs of trial $n$ ) was not explicitly modeled here, since activation values were reset at each trial. Instead, we simply evaluated whether the pattern of adaptation across conditions was a function of stimulus conflict or of response conflict on the previous trial. The adaptation (reduction) of the flanker effect as a function of conflict on the preceding trial in Verbruggen et al.'s data is reproduced in Figure 2A, and RT data are shown in Figure 2B. Figures 2C and 2D plot stimulus conflict and response conflict, respectively. Figure $2 \mathrm{E}$ shows the predicted RTs. According to the model, RTs are determined by the amount of response conflict. The observed RTs clearly follow the predicted pattern. On the other hand, adaptation of the flanker effect does not follow response conflict: The observed difference in adaptation is between Co and SI (where stimulus conflict comes in) and not between SI and RI (where response conflict comes in). It is clear that adaptation is sensitive to stimulus conflict and not to response conflict.

Although Verbruggen et al.'s (2006) data are encouraging with respect to the role of stimulus conflict in conflict adaptation, the predicted pattern of adaptation is still the same for both conflict measures (with conflict not decreasing as one proceeds from Co to SI to RI). For a strong argument for the role of stimulus conflict, we need a task in which stimulus conflict and response conflict maximally disagree. Moreover, it would be interesting to see how adaptation changes when stimulus conflict gradually increases.
A

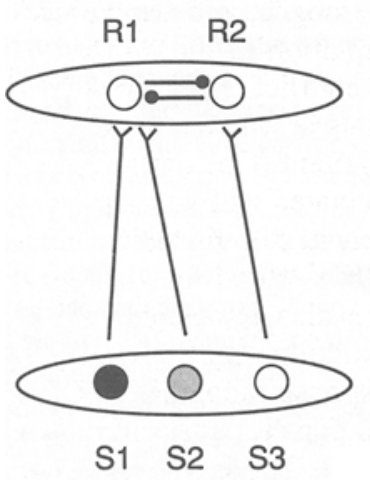

B

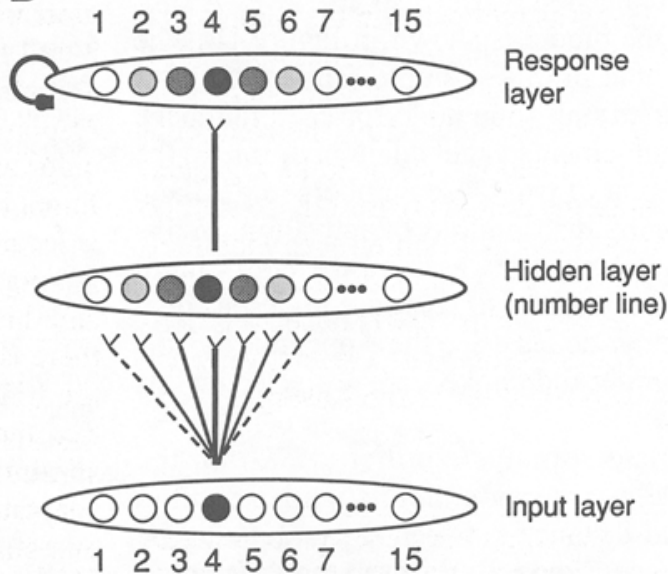

Figure 1. (A) The stimulus (S) conflict model. A stimulus-incongruent trial is presented in which the target activates $S 1$ (activation $=1$ ) and the flanker $S 2$ (activation $=0.5$ ), but both are associated with the same response. (B) The number model. For clarity, only the connections originating from the fourth input node and the fourth mental number line node are shown. The solidness of the line between two nodes is an indication of the strength of the connection between the two nodes. The response layer is fully interconnected with inhibitory connections. 
To achieve these goals, we used a numerical flanker task (Nuerk, Bauer, Krummenacher, Heller, \& Willmes, 2005; Ullsperger, Bylsma, \& Botvinick, 2005). A central target was flanked by two irrelevant numbers on each side, and participants had to respond to the central target by pressing the corresponding key on a numerical keypad. The advantage of this task is that only a small proportion of trials is eliminated when response repetitions, flanker repetitions, negative priming transitions, and target-become-flanker transitions are excluded (which is necessary to exclude bottom-up repetition effects). Ullsperger et al. observed an adaptation effect: After incongruent trials (i.e., when target and flankers are different), the flanker effect was reduced. They also observed a trend toward slower responses following incongruent trials, which was also interpreted as adaptive behavior following conflict.

The numerical flanker effect has the added advantage that stimulus and response conflict vary as a function of numerical distance between target and flanker. Nuerk et al. (2005) observed that when target and flankers were associated with different responses, the flanker effect decreased as the target-flanker distance increased. Similar parametric effects are also obtained in priming studies, including studies including tasks of number comparison (Naccache \& Dehaene, 2001), parity judgment and number naming (Reynvoet \& Brysbaert, 1999), and sequential (trial-to-trial) analyses (Koechlin, Naccache, Block, \& Dehaene, 1999). These findings consistently point toward mandatory access of a mental number line during number processing (Dehaene, 2004). On the mental number line, close numbers are represented by overlapping distributions of activations; for example, the activation distributions of the numbers 2 and 3 overlap more strongly than those of the numbers 2 and 6 . Accordingly, we measured stimulus conflict on this mental number line. The model that we used is based on that reported by Verguts, Fias, and Stevens (2005). In order to obtain response conflict, we included lateral inhibitory connections at the response level. A simplified version of the model is shown in Figure 1B. The number 4 is presented in this example. The lower level codes for the input stimuli (one node for each number). The hidden layer implements a mental number line in the sense that presentation of two close numbers (e.g., 3 and 4) leads to overlapping distributions of activation. In this task, there are as many responses as there are input stimuli (1-1 mapping), so the number of response nodes is equal to the number of input nodes. (See the Appendix for formal details.) Both target and flankers are projected on this mental number line.

When we calculate stimulus conflict $(1 / S D)$ on the mental number line, we measure more stimulus conflict when the numerical distance between the target and the flanker increases (see Figure 3C). With small distances (e.g., 11211), the activation on the number line will show activation only around the number 2 , whereas with larger distances (e.g., 77277) the activation on the number line will show two separate peaks of activation, resulting in stronger stimulus conflict. Response conflict, on the other hand, is strongest when the target-flanker distance is small (see Figure 3D). This dissociation enables us to test whether conflict adaptation follows stimulus conflict or response conflict. The predicted RTs are presented in Figure 3E. The reason why this model predicts shorter RT with increasing flanker-target distance is formally similar to the reason why the interactive activation model (McClelland \& Rumelhart, 1981) predicts a neighbor inhibition effect in word priming. When the relevant and irrelevant stimuli (in this case, target and flanker, respectively) are close together at a stimulus/semantic level, the target stimulus will tend to (partly) support the flanker response, leading to a stronger inhibition from the flanker response node toward the target response node, and thus a slower rise in activation of the target response node.

In our experiment, we calculated the flanker effect as a function of target-flanker distance on the previous trial. If the flanker effect is sensitive to stimulus conflict on the previous trial, it should decrease with increasing target-flanker distance. If, on the contrary, the flanker effect is sensitive to response conflict on the previous trial, it should increase with increasing target-flanker distance. Although all incongruent trials are both SI and RI, the opposite directions of the predictions across target-flanker distances enables us to investigate whether conflict adaptation follows stimulus conflict or response conflict.

\section{METHOD}

\section{Participants}

Fifteen volunteers ( 9 females and 6 males; average age $=$ 22 years) received $€ 5$ for participating in the experiment. They were unaware of the purpose of the experiment.

\section{Materials and Procedure}

The participants were tested on Pentium computers running E-Prime software. The stimuli consisted of one central target and two flankers on each side of it. The total array was approximately $1.2 \times$ $0.5 \mathrm{~cm}$. Stimuli were presented at the center of a computer display, approximately $50 \mathrm{~cm}$ from the participants. The participants had to react as quickly as possible to the central number by pressing the corresponding key on the numerical pad of a keyboard. The participants were instructed to start with the right index finger above the 5 key and to use this finger only. Stimuli remained on the screen until a response key was pressed. This started the response-stimulus interval $(800 \mathrm{msec})$. The number 5 was never used as a target or a flanker.

When targets and flankers are randomly chosen, small and large distances are unequally distributed. For instance, all target numbers in a stimulus configuration have distance-1 trials (e.g., 44344), but only the targets 1 and 9 can have distance-8 trials (e.g., 11911). In order to have a balanced number of small- and large-distance trials, we presented target-flanker combinations with distances 7 and 8 more often. Target-flanker combinations with distance 8 were presented nine times, and those with distance 7 were presented three times. All other combinations (distances 1-6) were presented once for each target in each block. For each target, an equal number of congruent and incongruent trials was presented. This resulted in 148 trials in each of the seven blocks. Tables 1 and 2 show the exact numbers of trials for each distance before and after errors and repetition trials were excluded.

We conducted two separate analyses in order to test our predictions on target-flanker distance of the previous trial and that of the current trial. Since our predictions are formulated in terms of (linear) trends, we analyzed the data with Lorch and Myers's (1990) regression analyses. In both analyses, we excluded target repetitions, flanker 
Table 1

Average Number of Trials per Subject for Each Current Distance Before and After Exclusion of Errors and Repetition Trials

\begin{tabular}{lccccccccc}
\hline & \multicolumn{8}{c}{ Current Distance } \\
\cline { 2 - 9 } \multicolumn{1}{c}{ Trials } & 0 & 1 & 2 & 3 & 4 & 5 & 6 & 7 & 8 \\
\hline All & 518 & 84 & 70 & 56 & 42 & 56 & 42 & 84 & 84 \\
Errors excluded & 501 & 81 & 67 & 54 & 41 & 54 & 40 & 82 & 81 \\
Repetitions and errors excluded & 396 & 55 & 44 & 36 & 29 & 35 & 25 & 47 & 42 \\
\hline
\end{tabular}

repetitions, target-become-flanker transitions, flanker-become-target transitions, errors, and trials following errors.

\section{RESULTS}

In our first analysis, we investigated the influence of target-flanker distance on RTs. The median RT per subject was taken as a function of target-flanker distance (Figure 2B). Note that distance 0 (e.g., 22222) is a congruent trial. In order to test the linear trend from distance 1 to distance 9, we calculated the slope for the observed RTs for distances 1-9. There was a negative slope of -9.08 $[t(14)=-5.13, p \leq .001]$. RTs decreased as the numerical target-flanker distance increased. This pattern is predicted by the model (Figure 3E) and follows the response conflict pattern (Figure 3D).

One could argue that RTs decrease with increasing distance because the targets 1 and 9 were presented more often than the other targets. We conducted a control experiment in order to test this hypothesis. In this experiment, 15 participants performed the same numerical flanker task, but all target-flanker pairs were presented equally often. This resulted in 122 trials, again in each of seven blocks. RTs decreased with increasing distance, and the slope (-9.32)
Table 2

Average Number of Trials per Subject by Previous Distance for Congruent and Incongruent Trials

\begin{tabular}{|c|c|c|c|c|c|c|c|c|c|}
\hline \multirow[b]{2}{*}{ Trials } & \multicolumn{9}{|c|}{ Previous Distance } \\
\hline & 0 & 1 & 2 & 3 & 4 & 5 & 6 & 7 & 8 \\
\hline \multicolumn{10}{|c|}{ All Trials } \\
\hline Congruent & 253 & 44 & 36 & 29 & 22 & 26 & 21 & 43 & 44 \\
\hline Incongruent & 269 & 39 & 34 & 27 & 19 & 29 & 21 & 40 & 40 \\
\hline \multicolumn{10}{|c|}{ Errors Excluded } \\
\hline Congruent & 243 & 43 & 34 & 28 & 22 & 26 & 21 & 43 & 43 \\
\hline Incongruent & 258 & 38 & 33 & 26 & 19 & 28 & 20 & 39 & 39 \\
\hline \multicolumn{10}{|c|}{ Errors and Repetition Triais Excluded } \\
\hline Congruent & 210 & 33 & 26 & 21 & 17 & 19 & 15 & 28 & 26 \\
\hline Incongruent & 188 & 22 & 18 & 14 & 11 & 16 & 10 & 19 & 14 \\
\hline
\end{tabular}

Note-The average target-flanker distance on the current trial is provided after errors and repetition trials were excluded. Congruent, distance 0 ; incongruent, distances $1-8$.

was significant $[t(14)=4.21, p \leq .001]$. Thus, although distances 7 and 8 were infrequent in this control experiment, a data pattern similar to that of the main experiment was observed. Large-distance trials were too infrequent in this control experiment to conduct the sequential analysis.

The second analysis dealt with the adaptation of the flanker effect (incongruent - congruent) as a function of distance on the previous trial. The results are shown in Figure 3A. There is a significant negative slope of -2.81 $[t(14)=-2.56, p \leq .01]$, which demonstrates that there is an inverse relationship between the target-flanker distance of the previous trial and the size of the congruency effect. In other words, conflict adaptation becomes stronger as target-flanker distance increases. ${ }^{2}$ This was predicted on the basis of the stimulus conflict measure (Figure 3C) but not on the basis of the response conflict measure (Fig-
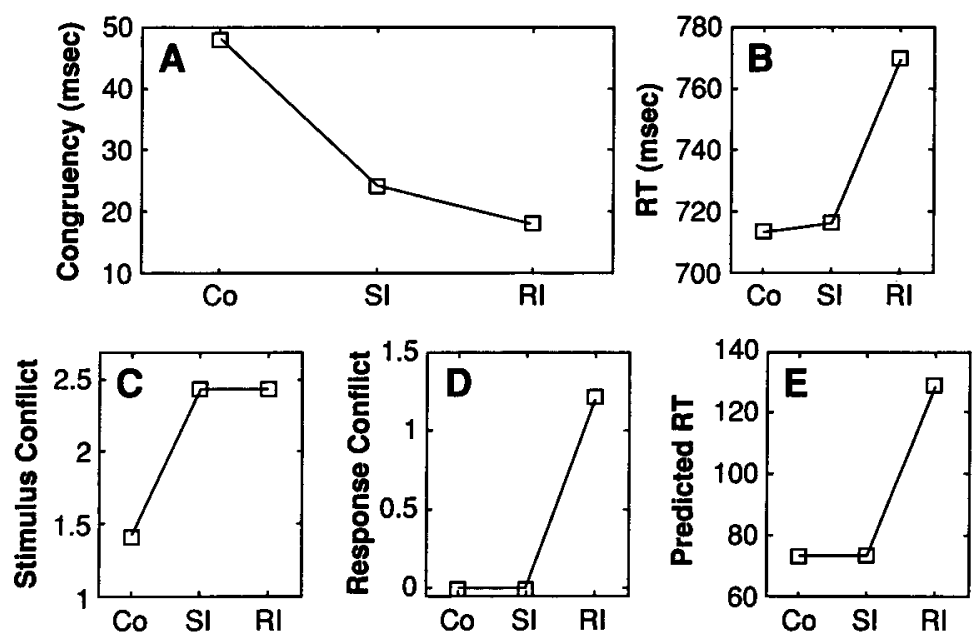

Figure 2. Panels $A$ and $B$ show data from Verbruggen et al. (2006). In panel A, the flanker effect (difference in response time [RT] between congruent and incongruent trials) is plotted as a function of distance on the preceding trial. In panel B, the observed RTs (in milliseconds) are plotted for congruent (Co), stimulus-incongruent (SI), and response-incongruent (RI) trials. Panels $\mathrm{C}$ and D show the outcomes of the stimulus and response confict measures, respectively, and panel E shows the predicted RTs. 

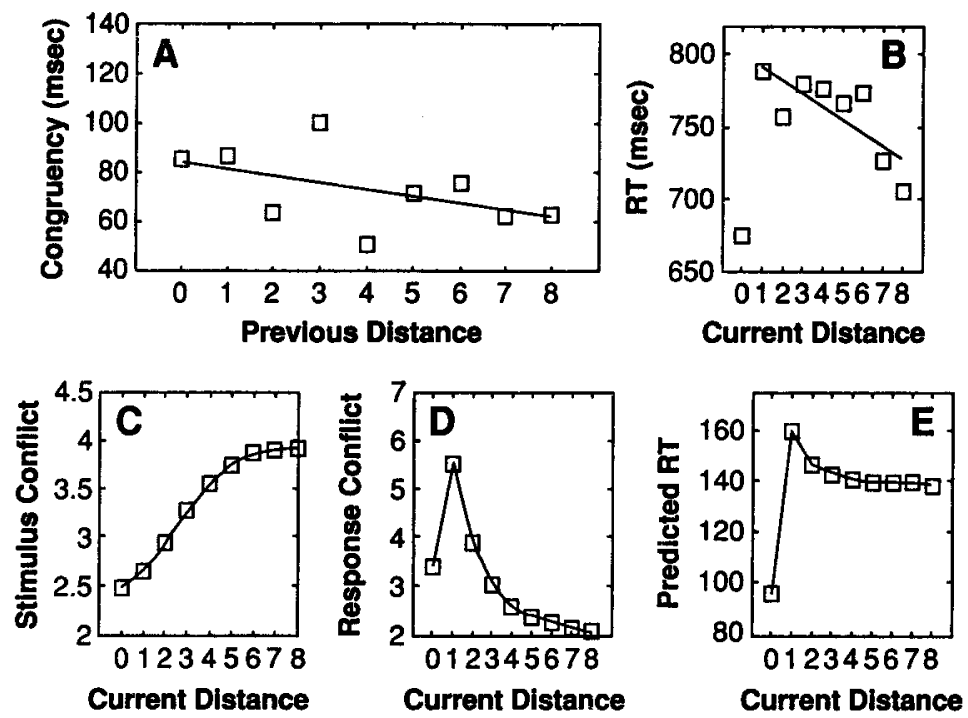

Figure 3. Panel A shows the observed flanker effect as a function of the previous target-flanker distance. Panel B shows the observed response times (RTs, in milliseconds) as a function of the target-flanker distance of the current trial. Panels C and D show the outcomes of the stimulus and the response conflict measures, respectively, and panel E shows the predicted RTs as a function of current target-flanker distance.

ure 3D). Finally, we note that RTs gradually increase with increasing previous distance [slope $=2.46 ; t(14)=1.85$, $p \leq .05$, one-tailed] (see also Ullsperger et al., 2005). This suggests that participants are more cautious with increasing previous distance and corroborates our interpretation of decreasing congruency as an adaptation effect.

\section{DISCUSSION}

Two important issues regarding cognitive control are centered on how the cognitive system detects conflict and how this information is used to improve information processing. Conflict monitoring theory (Botvinick et al., 2001) provides an elegant account for these issues. In the present context, it explains a very robust sequential effect in congruency tasks. The theory states that conflict detection on the preceding trial increases cognitive control, which reduces the congruency effect on the current trial. In the present study, we investigated the trigger behind this adaptation. Botvinick et al. argued that the trigger is response conflict. They introduced a measure of response conflict that works fine for many tasks. However, in most congruency tasks, response conflict is confounded with stimulus conflict. It is therefore important to investigate adaptation after pure SI trials. We have proposed a measure for stimulus conflict $(1 / S D)$ and investigated how both measures (stimulus conflict and response conflict) predicted effects of current and previous target-flanker distances in a numerical flanker task. In this task, stimulus conflict increased with increasing target-flanker distance, whereas response conflict decreased with increasing target-flanker distance (for all distances $\geq 1$ ), allowing us to dissociate the two types of conflict.
The RTs decreased as the current target-flanker distance increased. This is in line with the decreasing response conflict measure. In contrast, we observed decreasing congruency effects (adaptation) as previous-trial targetflanker distance increased. This demonstrates that conflict adaptation is sensitive not to the amount of response conflict, but rather to the amount of stimulus conflict. We also observed that overall RT increased with increasing preceding distance. This is in line with our adaptation interpretation: Adaptation results in both a higher response threshold (increasing RT) and a stronger focus on the relevant dimension (decreasing the congruency effect).

Other observations are also problematic for the traditional role of response conflict. In the Simon task, Burle, Allain, Vidal, and Hasbroucq (2005) observed on congruent trials that activation of the incorrect response sometimes leads to motor activity as measured by electromyographic activity (EMG). They argued that these congruent trials can be classified as conflict trials and consequently investigated adaptation after these trials. Although these congruent trials with incorrect EMG activation certainly qualify as response conflict trials, no adaptation was observed. This is consistent with our interpretation of the role of stimulus conflict and our own findings. Adaptation is triggered not by high levels of response conflict, but by high levels of stimulus conflict.

\section{REFERENCES}

Botvinick, M. M., Braver, T. S., Barch, D. M., Carter, C. S., \& CoHen, J. D. (2001). Conflict monitoring and cognitive control. Psychological Review, 108, 624-652.

Burle, B., Allain, S., Vidal, F., \& Hasbrouce, T. (2005). Sequen- 
tial compatibility effects and cognitive control: Does conflict really matter? Journal of Experimental Psychology: Human Perception \& Performance, 31, 831-837.

DEHAENE, S. (2004). The neural bases of subliminal priming. In N. Kanwisher \& J. Duncan (Eds.), Functional neuroimaging of visual cognition: Attention and performance XX (pp. 205-224). Oxford: Oxford University Press.

Gratton, G., Coles, M. G. H., \& Donchin, E. (1992). Optimizing the use of information: Strategic control of activation of responses. Journal of Experimental Psychology: General, 121, 480-506.

HOMMEL, B., PROCTOR, R. W., \& VU, K.-P. (2004). A feature-integration account of sequential effects in the Simon task. Psychological Research, 68, 1-17.

Kerns, J. G., Cohen, J. D., MacDonald, A. W., III, Cho, R. Y., StengER, V. A., \& CARTER, C. S. (2004). Anterior cingulate conflict monitoring and adjustments in control. Science, 303, 1023-1026.

Koechlin, E., Naccache, L., Block, E., \& Dehaene, S. (1999). Primed numbers: Exploring the modularity of numerical representations with masked and unmasked semantic priming. Journal of Experimental Psychology: Human Perception \& Performance, 25, 1882-1905.

KUNDE, W. (2003). Sequential modulations of stimulus-response correspondence effects depend on awareness of response conflict. Psychonomic Bulletin \& Review, 10, 198-205.

LORCH, R. F., JR., \& MYERS, J. L. (1990). Regression analyses of repeated measures data in cognitive research. Journal of Experimental Psychology: Learning, Memory, \& Cognition, 16, 149-157.

MAYR, U., AWH, E., \& LAUREY, P. (2003). Conflict adaptation effects in the absence of executive control. Nature Neuroscience, 6, 450-452.

MCClelland, J. L., \& RumelhaRT, D. E. (1981). An interactive activation model of context effects in letter perception: Part I. An account of basic findings. Psychological Review, 88, 375-407.

NACCACHE, L., \& DEHAENE, S. (2001). Unconscious semantic priming extends to novel unseen stimuli. Cognition, 80, 215-229.

Notebaert, W., Gevers, W., Verbruggen, F., \& Liefooghe, $B$. (2006). Top-down and bottom-up sequential modulations of congruency effects. Psychonomic Bulletin \& Review, 13, 112-117.

NotebaerT, W., Soetens, E., \& Melis, A. (2001). Sequential analysis of a Simon task: Evidence for an attention-shift account. Psychological Research, 65, 170-184.

Nuerk, H.-C., BaUer, F., KRUMmenaCHER, J., Heller, D., \& Willmes, K. (2005). The power of the mental number line: How the magnitude of unattended numbers affects performance in an Eriksen task. Psychology Science, 47, 34-50.

Reynvoet, B., \& Brysbaert, M. (1999). Single-digit and two-digit Arabic numerals address the same semantic number line. Cognition, 72, 191-201.

STÜrMER, B., LeUTHOLD, H., SOETENS, E., SCHRÖTER, H., \& SOMMER, W. (2002). Control over location-based response activation in the Simon task: Behavioral and electrophysiological evidence. Journal of Experimental Psychology: Human Perception \& Performance, 28, 1345-1363.

Ullsperger, M., BYLSMA, L. M., \& Botvinick, M. M. (2005). The conflict-adaptation effect: It's not just priming. Cognitive, Affective, \& Behavioral Neuroscience, 5, 467-472.

VAN Veen, V., \& CARTER, C. S. (2005). Separating semantic conflict and response conflict in the Stroop task: A functional MRI study. NeuroImage, 27, 497-504.

van Veen, V., Cohen, J. D., Botvinick, M. M., Stenger, V. A., \& CARTER, C. S. (2001). Anterior cingulate cortex, conflict monitoring, and levels of processing. Neurolmage, 14, 1302-1308.

VerbrugGen, F., Notebaert, W., Liefooghe, B., \& VANDierenDONCK, A. (2006). Stimulus- and response-conflict-induced cognitive control in the flanker task. Psychonomic Bulletin \& Review, 13, 328-333.

Verguts, T., Fias, W., \& Stevens, M. (2005). A model of exact smallnumber representation. Psychonomic Bulletin \& Review, 12, 66-80.

WüHR, P., \& ANSORGE, U. (2005). Exploring trial-by-trial modulations of the Simon effect. Quarterly Journal of Experimental Psychology, 58A, 705-731.

\section{NOTES}

1. The denominator used in calculating the (square of the) $S D$ was taken to be $1 / n$ rather than $1 /(n-1)$ because the $S D$ is not intended as a sample approximation to a population value.

2. In Table 2, we added the average distance for the incongruent trials after each distance. After distances 7 and 8 , the average target-flanker distance is smaller than it is after other distances. This is because after 11911 , all target-flanker combinations with 1 or 9 as target or flanker were excluded, except on large-distance trials. On the basis of this, one would expect larger flanker effects after distances 7 and 8 . The fact that we observed the opposite excludes current target-flanker distance as a possible confound. 
APPENDIX

\section{The Stimulus-Response Model}

We used the minimal stimulus-response configuration necessary to distinguish between congruent (Co), stimulus-incongruent (SI), and response-incongruent (RI) trials, which is a three-stimulus, two-response configuration. The relevant (target) stimulus has an activation value of 1 in its corresponding input node, and an irrelevant (flanker) stimulus has an activation value of 0.5 . Each value of the input vector $x$ corresponds to one stimulus, and the activation values add for stimulus. For example, if the stimulus 1 was used as both target and flanker, the input pattern of the network was $x=(1+0.5,0,0)=(1.5,0,0)$; if the stimuli 1 and 2 were used as target and flanker, respectively, the input pattern was $x=(1,0.5,0)$

The stimuli 1 and 2 mapped to response 1, and the stimulus 3 mapped to response 2 (see Figure 1A). The inhibitory connection between response nodes was equal to -0.2 (used in Equation 1 of the text). The net input to the response nodes was a combination of bottom-up and lateral input-specifically,

$$
\text { net }_{j}=\sum_{i} v_{i j} x_{i}+\sum_{i} w_{i j} z_{i},
$$

where $v_{i j}$ is the connection between input node $i$ and response node $j$. Activation values $z_{j}$ of response nodes were adapted as

$$
z(t+1)=z(t)+\delta \times \text { net } \times(M-z)
$$

if net $>0$, and as

$$
z(t+1)=z(t)+\delta \times \text { net } \times(z-m)
$$

otherwise (see McClelland \& Rumelhart, 1981). A response was given if the maximally active response node was sufficiently higher than the second most active response node (i.e., with difference $\geq \tau$ ). Here and in the number model, very similar results were obtained with the criterion that a response node should exceed a fixed threshold. The parameters $\delta, M, m$, and $\tau$ were set at $0.01,15,0$, and 10 , respectively. Small changes in these values led to similar results. Response conflict was measured as the average energy (Equation 1) from stimulus presentation to response.

\section{The Number Model}

The number model was based on the model of Verguts et al. (2005). As in that model, there were 15 input nodes, each coding for one number from 1 to 15 . As in the $S-R$ model, the relevant (target) stimulus had an activation value of 1 in its corresponding input node and an irrelevant (flanker) stimulus had an activation value of 0.5 . Activation values $x_{i}$ in the input layer were transformed to a "semantic" layer, or mental number line (see Figure 1B), according to

$$
y_{j}=\sum_{i} \exp (-\alpha \mid i-j) x_{i} .
$$

The parameter $\alpha$ was set at .7. Activation dynamics in the response layer were similar to those in the S-R model (see Equations A1-A3), except that input to the response nodes arrived from the semantic layer (i.e., activation values $y$ ). All parameter settings were the same as in the S-R model. 